\title{
$\varangle \quad$ Los archivos autonómicos en el censo del sistema andaluz de archivos
}

\author{
Mateo Antonio Páez García \\ Facultativo del Archivo General de Andalucia
}

Aunque frío y pesado, el censo del Sistema Andaluz de Archivos es un instrumento de información -como se define en el Proyecto de Reglamento del Sistema Andaluz de Archivos- de primer orden. No vamos a hablar de las vicisitudes y retrasos que ha sufrido este instrumento cuya creación estaba prevista desde 1984 (I), pero sí hemos de referirnos a su estructura, pues de entre el abanico de posibilidades que se abrían para elaborarlo - censar todos los archivos andaluces, censar parte de ellos- y de las diversas maneras en que se podía estructurar, la Directora del Archivo General de Andalucía y Coordinadora de los trabajos del censo, Antonia Heredia Herrera, eligió acotarlo y limitarlo a los centros del Sistema Andaluz, es decir, todos aquellos de competencia o gestión autonómica de titularidad pública y los de titularidad privada que reciban de los poderes públicos subvenciones o ayudas en cuantía superior a la mitad de su presupuesto ordinario o disfruten de beneficios fiscales (2), o simplemente soliciten formar parte del Sistema a la Consejería de Cultura (3). Quedaban, pues, excluidos archivos tan importantes como los universitarios o los de Justicia, o tan emblemáticos como el General de Indias, de titularidad y gestión estatal, o tan numerosos y ricos como los privados, incluyendo los eclesiásticos, y no integrados en el Sistema.

Dentro de este Sistema Andaluz de Archivos se articulan a su vez cuatro subsistemas definidos por la titularidad de los mismos: el subsistema de titularidad autonómica, el de titularidad estatal y gestión autonómica, el de titularidad local y el privado (4). Pero dentro del Sistema, el ciclo vital de los documentos y su paso de unos archivos a otros, tejen una serie de redes de centros, que para el caso del subsistema de archivos de titularidad autonómica, son dos:

- por un lado la red de los servicios centrales: archivos de oficina de los servicios centrales (Consejerías, Organismos Autónomos y Empresas Públicas), Archivos Centrales y Archivo General de Andalucía.

- por otro la red de servicios periféricos, con la particularidad de que los archivos definitivos de la documentación de las Delegaciones y Gerencias provinciales son los Archivos Históricos Provinciales, que en la actualidad son de titularidad estatal, aunque la Comu- nidad Autónoma tenga encomendada la gestión. Es decir, están involucrados en esta red dos susbsistemas.

Si acudimos a lo hasta ahora publicado, por ejemplo, el Anuario estadístico de Andalucía 1996, elaborado por el Instituto de Estadística de Andalucía, la situación, o la mera existencia, de los archivos de titularidad andaluza, es bastante confusa. En él aparecen 92 archivos dependientes de la Administración Autonómica, en un caos de cifras que va de I archivo en Huelva y Málaga, a 32 (i!) en Almería, pasando por 13 en Sevilla. Fuera de eso, parece que los únicos archivos que desarrollan actividades son los Históricos Provinciales.

Estas simples noticias no son de recibo; de ahí la necesidad absoluta de este censo, porque él, o mejor sus lecturas, desvelan una serie de datos, unas veces complacientes y otras no tanto -a veces nada- sobre los archivos de nuestra comunidad, pero absolutamente necesarios al profesional de archivos y a los responsables de los mismos y de la política archivística. Y todo ello sabiendo que la denominación de "archivo" es, en la mayoría de los casos, bastante generosa con la realidad. Por eso, la Coordinadora del censo ha seguido el criterio estricto de diferenciar unos archivos con un responsable al frente donde está garantizado el servicio -fin último de estos centros- de unos depósitos de archivo donde, con mayor o menor fortuna, se acumulan los documentos. Esto independientemente del volumen, antigüedad o riqueza documental que unos y otros custodien.

En estas líneas vamos a contemplar brevemente a la luz del censo el subsistema de archivos de titularidad autonómica. El nacimiento de este subsistema puede datarse en 1987, cuando se crea el Archivo General de Andalucía (5) como el primer Archivo propiamente andaluz (aunque su creación ya estaba prevista desde 1984 (6)). El Decreto de creación del Archivo General en su artículo 5 ya establecía que en todas las Consejerías existiese un archivo central dependiente funcionalmente de la Consejería de Cultura, pero no tendría plasmación legal hasta dos años más tarde, cuando por el Decreto 233/1989, de 7 de noviembre, se establece el funcionamiento de los archivos centrales de las Consejerías, Organismos Autónomos y Empresas de la Junta de Andalucía, como archivos que guardasen la documentación hasta su transferencia al Archivo General de Andalucía.

A más de siete años de estos planteamientos normativos, ¿cuál es la situación real que detecta el censo? Vayamos 
simplemente al directorio final que acompaña a estas páginas, o veamos el cuadro adjunto: frente a 28 archivos, 97 centros e instituciones andaluzas no cuentan con un archi-

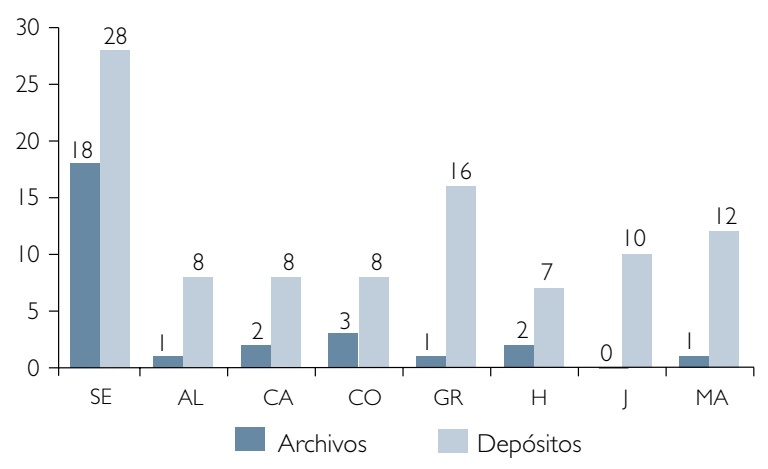

vo propiamente dicho.

Desde luego, hay instituciones y centros de escasísima producción documental que no necesitan de un profesional de archivos, pero otras lo están clamando a voces. El siguiente cuadro, refleja por orden los 22 archivos autonómicos con mayor volumen de documentación. Es muy esclarecedor que el primero de la lista no sea un archivo de servicios centrales, sino un archivo de Delegación.

Y decimos que es esclarecedor porque en el subsistema autonómico se ha descuidado la red periférica, a pesar del importantísimo volumen documental, parangonable, y a

ARCHIVO

\begin{tabular}{lll}
\hline Delegación Provincial de Hacienda & Sevilla & 2995,5 \\
\hline Archivo Central de la C. de Economía y Hacienda & Sevilla & 2875,0 \\
\hline Delegación Provincial de Industria, Comercio y Turismo & Sevilla & 2216,2 \\
\hline Delegación Provincial de Educación y Ciencia & Sevilla & 2216,0 \\
\hline Archivo Central de la C. de Educación y Ciencia & Sevilla & 2201,0 \\
\hline Delegación Provincial de Salud & Sevilla & 2118,2 \\
\hline Archivo Central de la C. de Obras Públicas y Transportes & Sevilla & 2100,0 \\
\hline Archivo Central de la C. de Gobernación & Sevilla & 2058,0 \\
\hline Archivo Central Instituto Andaluz de Servicios Sociales & Sevilla & 2044,0 \\
\hline Archivo General de Andalucía & Sevilla & 2000,0 \\
\hline Delegación Provincial de Trabajo y Asuntos Sociales & Córdoba & 1975,0 \\
\hline Delegación Provincial de Obras Públicas y Transportes & Córdoba & 1968,0 \\
\hline Delegación Provincial de Obras Públicas y Transportes & Sevilla & 1700,0 \\
\hline Delegación Provincial de Trabajo e Industria & Cádiz & 1520,0 \\
\hline Delegación Provincial de Trabajo & Sevilla & 1268,4 \\
\hline Delegación Provincial de Economía y Hacienda & Almería & 1250,6 \\
\hline Delegación Provincial de Obras Públicas & Jaén & 1231,0 \\
\hline Archivo Central del Servicio Andaluz de Salud & Sevilla & 1212,0 \\
\hline Delegación Provincial de Trabajo e Industria & Málaga & 1159,0 \\
\hline Delegación Provincial de Trabajo e Industria & Almería & 1121,8 \\
\hline Delegación Provincial de Obras Públicas y Transportes & Cádiz & 1053,0 \\
\hline Delegación Provincial de Economía y Hacienda & Granada & 1000,1 \\
\hline
\end{tabular}

veces superior, al de la red central: falta de personal técnico, carencia en las instalaciones, ausencia de servicio, son algunas de las situaciones que se detectan en muchos archivos de Delegaciones Provinciales (7). E insistimos en el volumen documental: algunas instituciones casi llegan a los tres kilómetros lineales de documentos, muchas superan los dos kilómetros. Para que nos hagamos una idea de lo que esto significa, baste tener en cuenta que una institución casi cinco veces centenaria, como la Chancillería de Granada, tiene $8.810 \mathrm{~m} / \mathrm{l}$ de documentación; los centros de la Administración autonómica han producido una cuarta parte ¡en el espacio de quince años!

Tocamos aquí el gran problema de los archivos administrativos y de sus archiveros, que no es, por paradójico que parezca, el de la conservación de la documentación, o para ser más precisos, el de su conservación indiscriminada, si no el de su selección, con la eliminación de lo inservible y la preservación permanente de lo que lo merezca (8). Pero para saber qué documentación se puede eliminar sin riesgos para la seguridad de la Administración y de los administrados es necesario llevar a cabo estudios de identificación y valoración de series, trabajo lento y laborioso que necesita de un personal de archivos por ahora escaso y volcado en otras tareas del archivo.

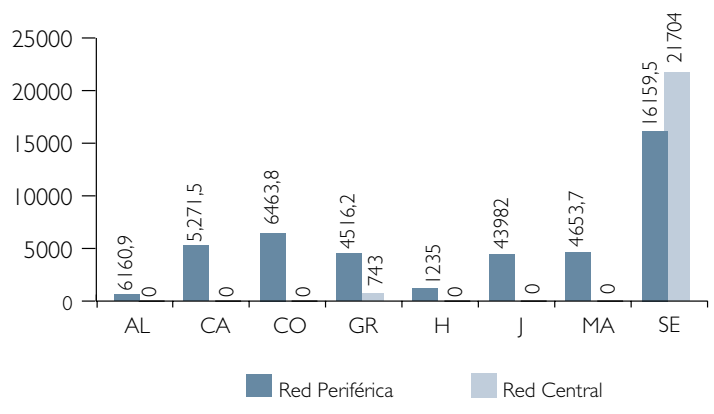

Pero hay otro segundo dato que destaca de esta escala. El centro cabecera del Sistema Andaluz, el Archivo General de Andalucía, ocupa sólo la décima posición por volumen documental, superado por cinco archivos centrales de Consejerías. Esto quiere decir que la documentación no cumple su ciclo vital y está quedando estancada indefinidamente en unos archivos contemplados como transitorios. La causa de este estancamiento enlaza, en parte, con la anterior: ausencia de una política de eliminación que impida estas enormes acumulaciones. Pero también hay carencias básicas en las instalaciones: recordemos que el Archivo General de Andalucía no cuenta aún con sede definitiva y sus depósitos están ya colapsados.

Aunque hasta ahora hayamos destacado lo sombrío, no queremos terminar dando una sensación de pesimismo, porque si es mucho lo que hay que avanzar en medios, en personal, es también muy importante la labor que están desarrollando en estos momentos los archiveros en pro de la conservación de esta parte primordial del Patrimonio Documental Andaluz como es la que produce la propia Administración autonómica. Ojalá el censo también contribuya a realzar el papel de estos profesionales de la información al servicio de los más "nuestros" de nuestros archivos: los autonómicos. 
ARCHIVOS DE TITULARIDAD AUTONÓMICA

\section{INSTITUCIÓN}

\section{SEVILLA}

1. Archivo General de Andalucía

2. Archivo Central de la Consejería Agricultura y Pesca

3. Archivo Central de la Consejería de Cultura

4. Archivo Central de la Consejería de Economía y Hacienda

5. Archivo Central de la Consejería de Educación y Ciencia

6. Archivo Central de la Consejería de Gobernación

7. Archivo Central de la Consejería de Medio Ambiente

8. Archivo Central de la Consejería de Obras Públicas y Transportes

9. Archivo Central de la Consejería de Presidencia

10. Archivo Central de la Consejería de Salud

11. Archivo Central de la Consejería de Trabajo e Industria

12. Archivo Central de la Consejería de Turismo y Deporte

13. Archivo Central del Instituto Andaluz de Servicios Sociales

14. Archivo Central del Servicio Andaluz de Salud

19. Archivo Central de la Emp. Pública de Suelo de Andalucía (EPSA)

30. Archivo Central de la Cámara de Cuentas de Andalucía

31. Archivo Central del Parlamento de Andalucía

34. Archivo Central de la Deleg. Prov. de Obras Públicas y Transportes
DIRECCIÓN

Almirante Apodaca, 2. Sevilla. Tf.: 4219193 4218248, 4227901 / Fax: 4214403

Juan de Lara Nieto. Sevilla. Tf.: 4552398

Carlos Cañal, 7. Sevilla. Tf.: 4564134

Avda. República Argentina, 21. Sevilla.

Tf.: 4555474 / Fax: 4238005

Avda. La Palmera, 41, y Avda. Manuel Siurot, s/n, y Avda. Rep. Argentina, 21 (sótano 01). Sevilla. Tf.: 4235188

Plaza Nueva, 4. Sevilla. Tf.: 4575500 / Fax: 4213819

Ronda de la Exposición, 53-posterior. Sevilla.

Tf.: 4460527 / Fax: 4460527

Plaza de la Contratación. Sevilla. Tf.: 4216315

Monsalves, 8 y 10. Sevilla. Tf.: 4218860

Avda. Innovación, Ed. Arena I. Sevilla.

Tf.: 4558126 / Fax: 4558173

Héroes de Toledo s/n. Sevilla. Tf.: 4555244 / Fax: 4555248

Avda. Rep. Argentina, 23. Sevilla. Tf: 4558411 / Fax: 4555700 Héroes de Toledo, s/n. Sevilla. Tf.: 4554043-41

Avda. de la Constitución, 18. Sevilla. Tf.: 4211602

Avda. Bonanza, 4, 2 ${ }^{\circ}$ Sevilla. Tf.: 4550270 / Fax: 4550255

Ramón y Cajal, 35. Sevilla. Tf.: 4662701

Andueza, 2. Sevilla. Tf.: 4592100 / Fax: 4592207

Carretera de Carmona, 13. Sevilla. Tf.: 4351236
RESPONSABLE

Antonia Heredia Herrera

F. Javier Fernández Sánchez.

Juan José Pérez Ruíz

Antonio Luis de Sara Jaramillo

María José de Trías Vargas.

Margarita Vila Pérez

Gerardo García León.

Eduardo Camacho Rueda

Luisa Fernanda de Juan y Santos

María Luisa Capote Gil.

Mercedes Pimentel Sánchez.

José Román Alvarez.

Rocío Luna Fernández-Arámburu

Reyes Pro Jiménez

Antonio García Rodríguez

Isabel Gómez de León Contreras

Antonio Serrano Cordón

Carlos Pery Seco

\section{ALMERÍA}

153. Archivo de la Delega. Prov. de Obras Públicas y Transportes* Hnos. Machado,4. Almería. Teléfono: 231099 / Fax: 264630

Amparo Balsells Fernández

Gracia García Moreno

\section{CÁDIZ}

271. Archivo de la Deleg. Provincial de Economía y Hacienda

272. Archivo de la Deleg. Provincial de Obras Públicas y Transportes
Plaza de España, 19. Cádiz. Teléfono: 245201 / Fax: 255302

Plaza de la Constitución s/n. Cádiz. Tel.: 256001 / Fax: 256500
María Dolores Gallardo Correa

Rosaura Gallego Durán

\section{CÓRDOBA}

327. Archivo de la Delegación Provincial de Gobernación

328. Archivo de la Deleg. Provincial de Obras Públicas y Transportes

329. Archivo de la Gerencia Provincial del IASS
San Felipe, 5. Córdoba. Teléfono: 471285 / Fax: 473222

Tomás de Aquino, 1. Córdoba. Tel.: 237509 / Fax: 235619

Avenida Ollerías, 43. Córdoba. Teléfono: 486410

\section{GRANADA}

433. Archivo del Patronato de la Alhambra y Generalife

Real de la Alhambra s/n. Granada. Tel.: 227525-26-27 / Fax: 226363

Esther Cruces Blanco

\section{HUELVA}

607. Archivo de la Delegación Provincial de Gobernación

608. Archivo de la Deleg. Provincial de Obras Públicas y Transportes
Sanlúcar de Barrameda, 3. Huelva. Tel.: 210300 / Fax: 210310 Rosa Ma Domínguez Domínguez José Nogales, 4. Huelva. Teléfono: 257909
Ana María Camacho Vallejo

Guillermina Moreno Muriel

Concepción León Herrera

\section{MÁLAGA}

811. Archivo de la Delegación de Obras Públicas y Transportes 
DEPÓSITOS DE ARCHIVOS DE TITULARIDAD AUTONÓMICA

\section{INSTITUCIÓN}

SEVILLA

15. Centro Andaluz de Arte Contemporáneo

16. Instituto Andaluz de la Mujer

17. Instituto Andaluz para la Administración Pública

18. Instituto de Estadística de Andalucía

20. Centro Transportes y Mercancías de Sevilla

21. Empresa Pública de Gestión de Programas Culturales

22. Empresa Pública de Puertos de Andalucía

23. E.P. Radio y Televisión de Andalucía

24. Empresa Pública de Gestión Medioambiental S.A. (EGMASA)

25. Empresa Pública para el Desarrollo Agrario y Pesquero de Andalucía, S.A. (DAP)

26. Instituto de Fomento de Andalucía (IFA)

27. INTURJOVEN

28. Pabellón de Andalucía, S.A. (PASA)

29. Verificaciones Industriales de Andalucía S.A.

32. Defensor del Pueblo Andaluz

35. Delegación Provincial Agricultura y Pesca

36. Delegación Provincial de Asuntos Sociales

37. Delegación Provincial de Cultura

38. Delegación Provincial Educación y Ciencia

39. Delegación Provincial de Gobernación

40. Delegación Provincial de Hacienda

41. Delegación Provincial Industria, Comercio y Turismo

42. Delegación Provincial de Medio Ambiente

43. Delegación Provincial de Salud

44. Delegación Provincial de Trabajo

45. Gerencia Provincial IASS

46. Gerencia Provincial EPSA

47. Gerencia Provincial del IFA

\section{DIRECCIÓN}

Levíes, $n^{0}$ 17. Sevilla. Tf: 4559875

Alfonso XII, nº 52. Sevilla. Tf: 4904800-080 / Fax: 4908393

María Auxiliadora, $n^{0} 13$. Sevilla. Tf: 4559600

Marqués de Nervión, $n^{0} 40$. Sevilla. Tf: 4559800

Crtra. Sevilla-Málaga Km. 4. Sevilla. Tf:

Ximénez de Enciso, 35. Sevilla. Tf: 4211256

San Gregorio, 7. Sevilla. Tf: 4560744

Ctra. San Juan-Tomares, km 1,3. San Juan de Aznalfarache. Tf: 5607851 / Fax: 5607687 Avenida de Las Acacias s/n. Sevilla. Tf: 4488400

Progreso, 20. Sevilla. Tf: 4232890

Torneo, 26. Sevilla. Tf: 49000164906300

Turia, 46. Sevilla. Tf: 4558293

Avenida de las Acacias. Sevilla. Tf: 4460465

Asunción, 24. Sevilla. Tf: 4282819

Reyes Católicos, 21. Sevilla. Tf: 4212121

Edificio Sevilla, 1. Sevilla. Tf: 4556300 / Fax: 4556336

Luis Montoto, 89. Sevilla. Tf: 5556060 / Fax: 4583155

Castelar, 22. Sevilla. Tf: 4559900 / Fax: 4559913

Ronda del Tamarguillo s/n. Sevilla. Tf: 4939100 / Fax: 4924161

Avda. de la Palmera, 24. Sevilla. Tf: 4249603 / Fax: 4249644

Albareda, 20 y Manuel Siurot. Sevilla. Tf: 4563672 / Fax: 4563984

Plaza de España-Puerta Navarra. Sevilla. Tf: 4232304-09 / Fax: 4232313

Avda. de la Raza s/n. Sevilla. Tf: 4230540 / Fax: 4230503

Luis Montoto, 89. Sevilla. Tf: 4556600 / Fax: 4556009

Blas Infante, Rep. Argentina. Sevilla (Sevilla). Tf: 4556060 / Fax: 4583155

Imagen, 12. Sevilla. Tf: 4216703 / Fax: 4211392

Avda. República Argentina, 24. Sevilla. Tf:

República Argentina, 50. Sevilla. Tf: 4459011 / Fax: 4459432

\section{ALMERÍA}

154. Delegación Provincial de Agricultura y Pesca

155. Delegación Provincial de Cultura

156. Delegación Provincial de Educación y Ciencia

157. Delegación Provincial de Economía y Hacienda

158. Delegación Provincial de Gobernación

159. Delegación Provincial de Medio Ambiente

160. Delegación Provincial de Salud

161. Delegación Provincial de Trabajo e Industria
Hermanos Machado, $n^{0} 4$, $3^{a}$ planta. Almería. Teléfono: 210111

Hermanos Machado s/n. Almería. (9) Teléfono: 237036-6945

Paseo de la Caridad, $n^{\circ}$ 125. Almería. Teléfono: 273211

Trajano, $n^{\circ}$ 13. Almería. Teléfono: 620200

Paseo de Almería, $n^{0} 74$. Almería. Teléfono: 213100

Centro Res. Oliveros Bl. Singular, $2^{\text {a }}$ pl. Almería. Teléfono: 237566

San Leonardo, $n^{0} 7$. Almería. Teléfono: 212700

Tiendas, $n^{0} 11$ y Hnos. Machado no 4 - 1 $^{\text {a }}$. Almería. Tel.: 271988 / Fax: 230822

\section{CÁDIZ}

273. Delegación Provincial de Agricultura y Pesca

274. Delegación Provincial de Cultura

275. Delegación Provincial de Educación

276. Delegación Provincial de Gobernación

277. Delegación Provincial de Medio Ambiente
Isabel la Católica, 8. Cádiz. Tf:

Isabel la Católica, 13. Cádiz. Tf: 212361 / Fax: 225701

Plaza de Mina, 18. Cádiz. Tf: 229158

Plaza de España, 19. Cádiz. Tf: 245200 / Fax: 252004

Avda. Ana de Viya, 3-3º. Cádiz. Tf: 274629 / Fax: 259419 
278. Delegación Provincial de Salud

279. Delegación Provincial de Trabajo e Industria

280. Delegación Provincial de Turismo y Deporte
María Auxiliadora. Cádiz. Tf: 250050

Muñoz Arenillas, s/n. Cádiz. Tf: 283011 / Fax: 256560

Plaza de España, 19. Cádiz. Tf: 245269 / Fax: 226533

\section{CÓRDOBA}

330. Delegación Provincial de Agricultura y Pesca

331. Delegación Provincial de Cultura

332. Delegación Provincial Economía y Hacienda

333. Delegación Provincial de Educación y Ciencia

334. Delegación Provincial de Industria, Comercio y Turismo

335. Delegación Provincial de Trabajo y Asuntos Sociales

336. Delegación Provincial del Servicio Andaluz de Salud

337. Conjunto Arqueológico Madinat al-Zahra
Tomás de Aquino, s/n. Córdoba. Tf: 237300

Samuel de los Santos Gener, 9. Córdoba. Tf: 454606

Ronda de los Tejares, 16. Córdoba. Tf: 480676 / Fax: 477697

Tomás de Aquino, s/n. Córdoba. Tf: 211700 / Fax: 236313

Tomás de Aquino, s/n. Córdoba. Tf:

Romero, 1. Córdoba. Tf: 298200

Avda. República Argentina, 34. Córdoba. Tf: 217500

Crtra. Palma del Río, KM.8. Córdoba. Tf: 329130 / Fax: 330595

\section{GRANADA}

418. Delegación Provincial de Agricultura y Pesca

419. Delegación Provincial de Asuntos Sociales

420. Delegación Provincial de Cultura

421. Delegación Provincial de Economía y Hacienda

422. Delegación Provincial de Educación y Ciencia

423. Delegación Provincial de Gobernación

424. Delegación Provincial de Medio Ambiente

425. Delegación Provincial de Obras Públicas y Transportes

426. Delegación Provincial de Salud

427. Delegación Provincial de Trabajo

428. Delegación Provincial de Turismo y Deporte

429. Gerencia Provincial del IASS

430. Centro Base de Minusvalías

431. Escuela Andaluza de Salud Pública

432. Gestión de Infraestructuras de Andalucía, S.A.

434. Consejo Consultivo de Andalucía
(Sin dirección). Granada. Tf:

Ancha de Gracia, 6. Granada. Tf: 250954-62 / Fax: 262836

Paseo de la Bomba, 11. Granada. Tf: 223411/2/6 / Fax: 225472

Tablas, 11-13. Granada. Tf: 249500 / Fax: 249596

Duquesa, 22. Granada. Tf: 242700/60

Gran Vía, 34. Granada. Tf: 205911

Cra. Sierra Nevada, km. 7. Granada. Tf: 248300

Avda. de la Constitución, 18. Granada. Tf: 282400/50 / Fax: 201912

Avda. del Sur, 13. Granada. Tf: 208211/50 / Fax: 273612

Plaza de la Trinidad, 11. Granada. Tf: 286450

Turismo: Plaza Isabel la Católica, 1. Tf: 227510.

Deporte: Paseo de la Bomba, 11. Granada. 223411/2.

Ancha de Gracia, 6. Granada. Tf: 250954/62

Ctra. de Alfacar, 13. Granada. Tf: 152366 / Fax: 289669

Campus Universitario de Cartuja. Granada. Tf: 161044 / Fax: 161142

Molinos, 65. Granada. Tf: 220695 / Fax: 228792

San Matías, 17. Granada. Tf: 221384 / Fax: 222887

\section{HUELVA}

609. Delegación Provincial de Agricultura

610. Delegación Provincial de Cultura

611. Delegación Provincial de Economía y Hacienda

612. Delegación Provincial de Educación y Ciencia

613. Delegación Provincial de Industria, Comercio y Turismo

614. Delegación Provincial de Salud

615. Delegación Provincial de Trabajo y Asuntos Sociales
Plus Ultra, 41. Huelva. Tf: 240611

Avenida Manuel Siurot, 6. Huelva. Tf: 257523

Plaza de la Constitución, 2. Huelva. Tf: 299400

Alameda Sundheim, 17. Huelva. Tf: 283011

Avenida de Alemania, 1. Huelva. Tf:

Martín Alonso Pinzón, 6. Huelva. Tf: 283625

La Fuente, 14. Huelva. Tf: 255016

\section{JAÉN}

700. Delegación Provincial de Agricultura y Pesca

701. Delegación Provincial de Asuntos Sociales

702. Delegación Provincial de Cultura

703. Delegación Provincial de Economía y Hacienda

704. Delegación Provincial de Educación y Ciencia
Avda. de Madrid, nº 25. Jaén. Tf: 223250 / Fax: 262208

Paseo de la Estación, nº 19. Jaén. Tf: 217500 / Fax: 217509

Martínez Montañés, no 8. Jaén. Tf: 295081 / Fax: 275439

Paseo de la Estación, no 19. Jaén. Tf: 215500 / Fax: 215523

Martínez Montañés, nº 8. Jaén. Tf: 295029 / Fax: 275437 
705. Delegación Provincial de Gobernación

706. Delegación Provincial de Medio Ambiente

707. Delegación Provincial de Obras Públicas

708. Delegación Provincial de Salud

709. Delegación Provincial de Trabajo e Industria
Plaza de las Batallas, $n^{\circ}$ 3. Jaén. Tf: 254716

Avda. Andalucía, $n^{\circ} 79,1^{\circ}$. Jaén. Tf: 215000

Sta. María del Valle s/n. Jaén. Tf: 211100 / Fax: 225652

Paseo de la estación, nº 15. Jaén. Tf: 222154 / Fax: 222864

Paseo de la Estación, nº 19. Jaén. Tf: 217500

\section{MÁLAGA}

812. Delegación Provincial de Agricultura y Pesca

813. Delegación Provincial de Asuntos Sociales

814. Delegación Provincial de Cultura

815. Delegación Provincial de Economía y Hacienda

816. Delegación Provincial de Educación y Ciencia

817. Delegación Provincial de Gobernación

818. Delegación Provincial de Medio Ambiente

819. Delegación Provincial de Salud

820. Delegación Provincial de Trabajo e Industria

821. Delegación Provincial de Turismo y Deportes

822. Empresa Pública Hospital Costa del Sol

823. Empresa Pública de Emergencias Sanitarias
Avenida de la Aurora. Málaga. Tf: 2329500

Manuel Agustín Heredia, nº 26. Málaga. Tf: 2210412-13

Avda. Herrera Oria, $n^{\circ} 88$. Málaga. Tf:

Avda. de la Aurora, $n^{0} 9$. Málaga. Tf:

Avda. Aurora s/n. Málaga. Tf: 2134700

Alameda, no 18 y Avda. Aurora, 57. Málaga. Tf: 2214820 / Fax: 2338149

Mauricio Moro, nº 2. Málaga. Tf: 2401130

Manuel A. Heredia, 34; Córdoba, 4. Málaga. Tf: 2224900

Manuel A. Heredia y Avda. Aurora. Málaga. Tf: 2215133

Avda. de la Aurora. Málaga. Tf: 2347300

Crtra. Nal. 340, km. 187. Marbella. Tf: 2862748 / Fax: 2823219

Avenida Juan López Peñalver. Camanillas (Málaga). Tf: 2626450

\section{Notas:}

I. A ello se ha referido en este mismo Boletín HEREDIA HERRERA, Antonia, "Aproximación al Patrimonio Documental: el Censo del Sistema Andaluz de Archivos", PH Boletín del Instituto Andaluz de Patrimonio Histórico, en prensa.

2. Según el art. II de la ley 3/1984, de 9 de enero.

3. Así lo dispone el art. I I del Reglamento de Organización del Sistema Andaluz de Archivos y desarrollo de la Ley $3 / 1984$, de 9 de enero, aprobado por Decreto 73/1994, de 29 de marzo (BOJA nº 70, de 29 de marzo).

4. La Comunidad Autónoma Andaluza tiene competencia exclusiva, según el art. 13 de la citada Ley 3/1984, sobre el Archivo General de Andalucía, archivos de Diputaciones Provinciales andaluzas, archivos municipales andaluces y cualquier otro que no sea de titularidad estatal. A ellos se añaden los Archivos Históricos Provinciales y el de la Real Chancillería, de titularidad estatal, cuya gestión fue transferida por Real Decreto 864/1984, de 29 de febrero (BOJA n 57, de 8 de junio; corrección de errores en BOJA n 7, de 28 de enero de 1985; $\mathrm{BOE} \mathrm{n^{ \circ }}$ । I3, de II de mayo; corrección de errores en $\mathrm{BOE} \mathrm{n}^{\circ} 161$, de 6 de julio), transferencia que se hizo efectiva por sendos convenios entre el Ministerio de Cultura y la Junta de Andalucía, de 9 de octubre de 1984 y de 23 de mayo de 1994 (este último publicitado por resolución de 27 de mayo de 1994 de la Dirección General de Bienes Culturales, BOJA nº 90, de 16 de junio, corrección de errores en BOJA n I07, de 14 de julio; $\mathrm{BOE}$ $n^{\circ} 147$, de 21 de junio).
5. Decreto 323/1987, de 23 de diciembre, por el que se crea el Archivo General de Andalucía (BOJA n 9, de 5 de febrero).

6. Ley $3 / 1984$, de 9 de enero, de Archivos (BOJA n ${ }^{\circ} 4$, de 10 de enero, corrección de errores en $\mathrm{BOJA} \mathrm{n}^{\circ} \mathrm{I}$, de 28 de nero; $\mathrm{BOE} \mathrm{n}^{\circ} 25$, de 30 de enero)

7. Por poner un ejemplo, en la mayoría de ellas las unidades de instalación están sin numerar, y en algunas que no citaremos,ni siquiera se ha podido cumplimentar la encuesta y sus datos aparecen en blanco en el censo

8. En un porcentaje muy alto, esa documentación es totalmente inútil cuando transcurre su vigencia administrativa. En países como los Estados Unidos sólo se conserva indefinidamente entre el 5 y el 10\% de la documentación producida.

9. El depósito de la Delegación alberga, además de los documentos, material arqueológico y libros del Centro Coordinador de Bibliotecas. La biblioteca y el museo carecen de depósito dedicado exclusivamente a Archivo. 